\section{Avaliação da assistência farmacêutica à gestante na rede básica de saúde do Município de Praia Grande, São Paulo, Brasil}

\author{
Assessment of pharmaceutical care for pregnant \\ women treated in the public health system \\ in Praia Grande, São Paulo State, Brazil
}

\author{
${ }^{1}$ Universidade Católica de \\ Santos, Santos, Brasil. \\ 2 Centro de Estudos de \\ Cultura Contemporânea, São \\ Paulo, Brasil. \\ Correspondência \\ A. Bousquat \\ Centro de Estudos de Cultura \\ Contemporânea. \\ Rua Airosa Galvão 64, \\ São Paulo, SP \\ 05002-070, Brasil. \\ aylene.bousquat@pesquisador. \\ cnpq.br
}

\begin{abstract}
The use of medication in primary health care has increased steadily in recent decades, thus highlighting the relevance of assessing the pharmaceutical care provided to patients in the primary care setting. This article aimed to evaluate the pharmaceutical care provided by the public system in Greater Metropolitan Santos, São Paulo State, Brazil, focusing specifically on women during gestation. Methods included secondary data analysis, document analysis, interviews with key players, and visits to health services and to the pharmaceutical supply center. Deficiencies were identified in most stages of pharmaceutical care: selection, planning, purchase, storage, distribution, and dispensing. The results suggest that in order to improve primary health care, with better access, equality, and comprehensiveness, the evaluation of pharmaceutical care should be incorporated as a routine procedure.
\end{abstract}

Pharmaceutical Care; Primary Health Care; Health Services Evaluation; Prenatal Care
Marlene Rosimar da Silva Vieira 1

Paulo Angelo Lorandi 1

Aylene Bousquat 1,2

\section{Introdução}

O uso de medicamentos na atenção primária à saúde vem crescendo continuadamente nas últimas décadas 1, de tal modo que avaliar a assistência farmacêutica prestada aos pacientes neste nível de atenção adquire relevância. Assumindose o referencial teórico para avaliação da atenção primária elaborado por Starfield 2, assistência farmacêutica comporia a dimensão da acessibilidade, que pode ser definida como presença ou ausência de barreiras financeiras, organizacionais e/ou estruturais para se conseguir atenção primária à saúde.

Acesso, no caso específico dos medicamentos, significa ter o produto adequado, para uma finalidade específica, na dosagem correta, pelo tempo que seja necessário, com a garantia da qualidade e a informação suficiente para o uso racional. A falta de acesso a medicamentos no nível primário da atenção onera ainda mais o sistema de saúde ao acarretar internações desnecessárias e o agravamento de casos clínicos 3 .

Lugones ${ }^{4}$, ao analisar as políticas de medicamentos do MERCOSUL, evidenciou a existência de enorme desperdício relacionado ao uso de medicamentos, estimando que $70 \%$ dos seus gastos são perdidos devido a preços e qualidade inadequados, armazenamento incorreto, expiração da validade, prescrição inadequada e falta de adesão ao tratamento. 
A análise da qualidade da assistência farmacêutica demanda a investigação de todas as suas fases constitutivas, quais sejam, atividades de seleção, programação, aquisição, armazenamento, distribuição e utilização do medicamento 5,6.

A seleção é realizada mediante conhecimento dos problemas de saúde prevalentes, identificando tratamentos de escolha, selecionando medicamentos e suas apresentações e decidindo que medicamento deve estar disponível em cada nível de atenção. Deste modo, é um elemento básico do sistema de abastecimento, é um passo importante na racionalização do uso e do manejo operativo, facilitando os trabalhos de planejamento, distribuição e armazenagem. A programação deve ser entendida como um conjunto de atividades que têm por objetivo determinar as necessidades de medicamentos dentro dos recursos disponíveis e garantir o acesso da população em quantidade e qualidade. A aquisição envolve a escolha do método de compra e o estabelecimento dos termos de contrato, assegurando a qualidade e garantindo o cumprimento dos seus termos. O armazenamento inclui controle de estoque e administração dos depósitos. A distribuição envolve o suprimento dos medicamentos às unidades de saúde. O uso está relacionado ao diagnóstico, à prescrição, à dispensação e ao consumo de medicamentos pelo paciente 5 .

Desse modo, a presente investigação tem por objetivo avaliar a assistência farmacêutica, em seus aspectos referentes à seleção, programação, aquisição, armazenamento e dispensação, ofertada na rede básica de saúde em município da Região Metropolitana da Baixada Santista, Estado de São Paulo, Brasil, delimitando-se para esta análise a saúde da mulher, especialmente no período gestacional. Tal delimitação decorre da constatação de que esta região vem apresentando seguidamente elevados coeficientes de mortalidade infantil, especialmente o componente neonatal, contrastando com indicadores sociais de seus municípios; ademais o "Pacto de Saúde" proposto pelo Ministério da Saúde 7 elenca no seu "Pacto pela Vida" a redução da mortalidade infantil e materna, como uma das seis prioridades nacionais na área da saúde. O Município de Praia Grande foi escolhido tanto por apresentar elevados coeficientes de mortalidade infantil e perinatal, tomando-se por referência a média estadual, quanto pela existência de dispensação regular de medicamentos na ampla rede básica de atenção à saúde existente (uma unidade para menos de 14.500 habitantes e 36 equipes de saúde da família em 2004) 8 , fornecendo uma situação propícia para a avaliação da assistência farmacêutica à gestante no nível primário de atenção à saúde.
Recorde-se que a atenção à saúde da mulher está fundamentada na Política Nacional de Atenção Integral à Saúde da Mulher (PNAISM), que aponta como um de seus objetivos específicos a criação de condições para "promover a atenção obstétrica e neonatal, qualificada e humanizada" 9 (p. 69). Porém, a realidade ainda se caracteriza por uma situação injusta e desigual no acesso da população aos medicamentos essenciais em nível de atenção primária.

\section{Métodos}

Foi realizado estudo no Município de Praia Grande, São Paulo, integrante da Região Metropolitana da Baixada Santista, com população fixa de 193.582 habitantes e população flutuante de 300 mil nos finais de semana chegando a 1.500 .000 na alta temporada. Seus coeficientes de mortalidade infantil variaram entre 17,77 e 25,6 mortes/mil nascidos vivos, entre 2001 e 2005, com predomínio do componente neonatal, ou seja, crianças que morreram até os 28 dias de vida, cujo comportamento é fortemente influenciado pela atenção oferecida pelos serviços de saúde. No tocante à mortalidade perinatal, que inclui os óbitos fetais a partir da 22a semana de gestação e os óbitos neonatais até o sexto dia de vida, o padrão é semelhante com coeficientes variando entre 15,89 a 28,19/mil nascidos vivos ou mortos, indicando mais uma vez uma assistência deficitária ao pré-natal e ao parto. Apresentou uma acelerada expansão do Programa Saúde da Família (PSF) nos últimos anos, passando de uma cobertura residual para mais de $50 \%$ da população em quatro anos. Esse ritmo de implantação não foi comum em outros municípios do mesmo porte e é único nesta região metropolitana 8,10.

Em todas as unidades básicas e de saúde da família e na Central de Abastecimento Farmacêutico foram realizadas análises de dados secundários, análise documental, entrevistas com atoreschave e observação com auxílio de roteiro.

O "padrão ouro" para a análise da qualidade da assistência farmacêutica foi construído baseado nas recomendações da Política Nacional de Medicamentos 11, no documento patrocinado pela Organização Pan-Americana da Saúde (OPAS) e Organização Mundial da Saúde (OMS) sobre gestão farmacêutica 5 e no manual técnico do Ministério da Saúde para atenção à gestante 12 .

Para a avaliação da seleção, a Relação Nacional de Medicamentos Essenciais (RENAME) 13 foi utilizada como referência por traduzir o resultado de estudos sobre as opções de medicamentos mais seguros e eficazes disponíveis no mercado brasileiro. Para as necessidades específicas das 
gestantes, optou-se, como padrão, pela relação de medicamentos constantes do documento Pré-Natal e Puerpério: Atenção Qualificada e Humanizada-Manual Técnico ${ }^{12}$.

Os critérios de qualidade para um bom armazenamento e dispensação dos medicamentos foram baseados no documento Assistência Farmacêutica para Gerentes Municipais 5.

Com base neste "padrão ouro" foram elaborados dois roteiros de observação, sendo um deles destinado para as unidades básicas de saúde $(n=7)$ e de saúde da família $(n=13)$, e outro para a Central de Abastecimento Farmacêutico. Após a assinatura do Termo de Consentimento Livre e Esclarecido, foram também realizadas entrevistas semi-estruturadas, com conteúdos distintos, com os funcionários responsáveis pela farmácia das unidades e, na Central de Abastecimento Farmacêutico, com o farmacêutico responsável.

As entrevistas e observações ocorreram no período de março a maio de 2006, após treinamento da equipe formada por docentes e acadêmicos do curso de farmácia e do mestrado em saúde coletiva da Universidade Católica de Santos.

O plano de análise das entrevistas e dos documentos consistiu na leitura compreensiva e exaustiva do material com a finalidade de se obter uma visão de conjunto, seguida da separação dos trechos mais marcantes. A seguir, tomando-se por referência o "padrão ouro" elaborado, esses trechos foram classificados possibilitando uma síntese dos mesmos.

\section{Resultados}

Os resultados apurados e suas conseqüentes análises serão apresentados na seqüência dos elementos constitutivos da assistência farmacêutica, a saber, seleção, programação e aquisição, armazenamento e dispensação (distribuição e uso) do medicamento 5 .

Na Tabela 1, foram relacionados alguns elementos que possibilitam caracterizar a qualidade da assistência farmacêutica prestada no que se refere à seleção, programação e aquisição 5,14.

A lista de padronizados (Tabela 2) da Secretaria de Saúde apresenta 253 medicamentos diferentes, e alguns desses medicamentos apresentam o mesmo princípio ativo, variando-se dosagem ou via de administração. Assim, em relação aos princípios ativos, a lista é composta por 168 substâncias diferentes.

Ao detalhar-se a composição da relação municipal de medicamentos, observa-se a ausência de 19 medicamentos preconizados pelo Ministério da Saúde para a garantia de uma adequada assistência farmacêutica à gestante 12 .

\section{Armazenamento}

Em visita à Central de Abastecimento Farmacêutico, foram identificadas situações (Tabela 3) que podem ser avaliadas como adequadas ou não, segundo normatização do Ministério da Saúde 14.

Na etapa da dispensação, o foco da análise recai na ponta do sistema, ou seja, nas unidades de atenção primária à saúde, sejam elas as tradicionais unidades básicas de saúde ou as unidades

Análise das etapas de seleção, programação e aquisição de medicamentos usando como referencial o "padrão ouro".

\begin{tabular}{lc}
\hline Etapas & "Padrão ouro" \\
\hline Existência da Comissão de Farmácia e Terapêutica & Não \\
Sistematização de procedimentos de padronização de medicamentos & Não \\
Critérios técnicos científicos para inclusão de medicamentos na lista & Não \\
RENAME como referencial de padronização & Não \\
Distribuição de todos os medicamentos essenciais nas unidades & Não \\
Atualização periódica da lista de medicamentos padronizados & Não \\
Padronização específica para gestante, parto e neonato & Não \\
Atendimento à legislação vigente das licitaçães públicas * & Sim \\
Efetivação de compras consolidadas & Não \\
Manutenção da regularidade no abastecimento & Não \\
Programação baseada em vários critérios técnicos & Não \\
\hline
\end{tabular}

RENAME: Relação Nacional de Medicamentos Essenciais.

*Lei $n^{\circ} .8 .66619$ 
Análise comparativa da lista de medicamentos padronizados pelo Município de Praia Grande, São Paulo, Brasil, referentemente à Relação Nacional de Medicamentos Essenciais (RENAME) 12 e ao manual técnico do Ministério da Saúde para a atenção à gestante 11.

\begin{tabular}{lcc}
\hline & Praia Grande & RENAME \\
\hline Número total de princípios ativos & 168 & 327 \\
Número total de especialidades & 253 & 520 \\
Total de preservativos & 3 & 2 \\
Dispositivos femininos de contracepção & 67 & 7 \\
Porcentagem de medicamentos essenciais para atenção à gestante & 21 \\
Número de medicamentos essenciais à gestante disponível em outras unidades & - \\
que não unidades básicas de saúde e unidades de saúde da família & -
\end{tabular}

Tabela 3

Avaliação das condições de armazenamento usando como referencial o "padrão ouro" 13.

\begin{tabular}{|c|c|}
\hline Condições de armazenamento & "Padrão ouro" \\
\hline Ausência de poeira no ambiente & Sim \\
\hline Ausência de mofo e sujidades nas paredes & Não \\
\hline Embalagens limpas dos medicamentos & Não \\
\hline Sem indícios de presença de roedores e aves & Sim \\
\hline Sem indícios de presença de insetos & Não \\
\hline Medicamentos fora de contato com o piso & Sim \\
\hline Medicamentos fora do contato direto com a parede & Não \\
\hline Coletores de lixo com tampas & Sim \\
\hline Presença de extintor de incêndio & Sim \\
\hline Registros diários de controle de temperatura & Sim \\
\hline Sistema de refrigeração adequado & Não \\
\hline Medicamentos com datas de validade mais recentes sendo distribuídos primeiro & Sim \\
\hline Controle de estoque informatizado & $\operatorname{Sim}$ \\
\hline Ventilação e luminosidade adequada & Sim \\
\hline Fácil acesso & Sim \\
\hline Dimensão adequada & $\operatorname{Sim}$ \\
\hline Sistema de vedação eficiente contra água da chuva & Não \\
\hline Armazenamento exclusivo de medicamentos & Não \\
\hline Procedimento operacional padrão para as atividades de armazenamento & Não \\
\hline Processo padronizado para a destruição de medicamentos vencidos & Não \\
\hline Restrição de acesso ao almoxarifado & Não \\
\hline
\end{tabular}

da saúde da família. Na Tabela 4, são visualizados os resultados encontrados correspondentes à estrutura organizacional da dispensação de medicamentos.

Durante as entrevistas com os funcionários responsáveis pela dispensação, alguns comentários espontâneos foram feitos e, de forma interessante, foram recorrentes em mais de uma unidade. Um dos motivos alegados para o constante rodízio de funcionários na dispensação é o fato de, ao trabalhar na farmácia, os funcionários poderiam ter um ritmo menos intenso de trabalho. Assim sendo, consideravam justo que todos pudessem usufruir desse benefício.

Outra observação feita pelos funcionários é que "não havia falta de medicamentos, exceto na 
Indicadores da qualidade de dispensação de medicamentos encontrados na rede básica do Município de Praia Grande, São Paulo, Brasil.

\begin{tabular}{|c|c|c|c|c|}
\hline \multirow[t]{2}{*}{ "Padrão ouro" } & \multicolumn{2}{|c|}{ Sim } & \multicolumn{2}{|c|}{ Não } \\
\hline & $\mathrm{n}$ & $\%$ & $\mathbf{n}$ & $\%$ \\
\hline Presença de farmacêutico & 0 & 0,0 & 20 & 100,0 \\
\hline Profissional fixo responsável pela dispensação de medicamentos & 2 & 10,0 & 18 & 90,0 \\
\hline Funcionários treinados para atuar com medicamentos & 0 & 0,0 & 20 & 100,0 \\
\hline Controle de estoque informatizado & 6 & 30,0 & 14 & 70,0 \\
\hline Restrição à entrada de pessoas no recinto da farmácia & 5 & 25,0 & 15 & 75,0 \\
\hline Recebimento de medicamento vencido & 2 & 10,0 & 18 & 90,0 \\
\hline Perda de medicamentos devido à validade vencida & 7 & 35,0 & 13 & 65,0 \\
\hline Disposição na ordem correta de vencimento & 20 & 100,0 & 0 & 0,0 \\
\hline Relato de ocorrência de falta de medicamentos para gestantes & 17 & 85,0 & 3 & 15,0 \\
\hline Ácido fólico & 13 & 65,0 & 7 & 35,0 \\
\hline$\alpha$-metildopa & 6 & 30,0 & 14 & 70,0 \\
\hline
\end{tabular}

última semana do mês". Como solução, os atendentes recomendavam ir a outras unidades, que também relatavam a mesma falta, levando os munícipes à "peregrinação" pelas diversas unidades de saúde.

\section{Discussão}

Estudos que visam avaliar a assistência farmacêutica durante o pré-natal são escassos. Na sua maioria, descrevem características da atenção médica, tais como número de consultas pré-natais e tipos de parto 15,16 .

$\mathrm{Na}$ análise da etapa da seleção de medicamentos (Tabela 1), no presente estudo, não foi encontrado no Município de Praia Grande um procedimento sistematizado e escrito dos seus critérios. Há uma lista impressa de medicamentos padronizados, a qual foi revisada em 2003, sem data precisa e nem processo escrito. $\mathrm{O}$ fato se mostra em oposição ao que preconiza o Ministério da Saúde, ou seja, para iniciar-se uma seleção de medicamentos, a primeira providência a ser tomada é a criação de uma Comissão de Farmácia e Terapêutica. A Comissão de Farmácia e Terapêutica deve ser uma instância colegiada, de caráter consultivo e deliberativo, que tem por finalidade assessorar ao gestor e equipe de saúde em assuntos referentes a medicamentos. Ressalta-se que a Comissão de Farmácia e Terapêutica deverá ser constituída por meio de instrumento legal, a fim de legitimar o processo 14 .

Como não existe uma Comissão de Farmácia e Terapêutica no município responsável por atu- alizações periódicas baseadas em critérios técnico-científicos, novas inclusões de medicamentos na lista de padronizados são realizadas quando os médicos a solicitam. A decisão sobre a inclusão dos medicamentos assume um caráter predominantemente administrativo, resultado da análise da disponibilidade de verbas. Desse modo, as inclusões de novos medicamentos acontecem sem estudos das evidências científicas que garantam a racionalidade de sua opção. Essa lógica de funcionamento amplifica as possibilidades de influência das indústrias farmacêuticas, as quais podem oferecer seus medicamentos e/ou equipamentos diretamente aos médicos, que, por sua vez, podem funcionar como elemento de pressão nas estruturas das secretarias municipais de saúde. Os critérios específicos para inclusão e exclusão de medicamentos da lista de padronizados deveriam estar normatizados em um formulário terapêutico 5 .

Um dos critérios para a inclusão de medicamentos deveria ser sua presença na RENAME 13 , uma vez que sua presença nessa lista garante maior conhecimento das vantagens e riscos do uso desses medicamentos. O Município de Praia Grande conta com uma lista de padronizados que contém 168 princípios ativos diferentes, em 253 especialidades (Tabela 2). Na lista, $75 \%$ dos princípios ativos são coincidentes com a RENAME. O número de padronizados pelo município é bastante inferior à RENAME (327 princípios ativos), diferentemente, por exemplo, da Relação Municipal de Medicamentos Essenciais do Município de São Paulo (REMUME São Paulo; 381 princípios ativos) 17 . 
Os medicamentos essenciais, como política defendida na Política Nacional de Medicamentos 11, devem ser acessíveis à população, de um modo geral, como forma de se garantir a racionalidade da farmacoterapia, bem como é uma forma de se minimizar os custos do tratamento. Para Guerra Jr. et al. 18, que estudaram o acesso aos medicamentos essenciais em municípios mineiros com índice de desenvolvimento humano (IDH) baixos (inferior a 0,699), constataram que a principal fonte de medicamentos essenciais é a farmácia comunitária privada, achado coerente com o de outros países como Gana, Camboja, Tanzânia, Senegal, Índia, Gana e El Salvador. Os autores defendem então a necessidade premente de se promover o conceito da essencialidade em todo o país para que se possa garantir maior acesso a um número cada vez maior de medicamentos essenciais.

Assim, o número restrito de medicamentos padronizados em Praia Grande, aliado à falta de critérios adequados para a seleção de medicamentos, indica que a assistência farmacêutica neste quesito é inadequada.

A padronização para gestante, em Praia Grande, não sofre nenhum tipo de critério específico. Na lista há, somente, $67 \%$ dos medicamentos preconizados pelo Ministério da Saúde para um bom atendimento à gestante 12 . Esse número pode ser reputado como insuficiente, já que o conceito de essencialidade dos medicamentos deve ser plenamente desenvolvido no país 11,18. Como o acesso aos medicamentos é por nível de atendimento, inclusive os referenciados pelo Ministério da Saúde (Tabelas 1 e 2), a gestante diabética, atendida em uma unidade básica, precisa deslocar-se em seu atendimento pré-natal para unidades diferenciadas para obter, por exemplo, a insulina.

Podemos ressaltar, ainda, que a REMUME São Paulo introduziu o fator de risco fetal para os medicamentos relacionados, o que permite "aumentar a qualidade do uso de medicamentos em gestantes” 17 (p. 17). Há de se considerar que o uso de medicamentos durante a gravidez pode ser necessário e é natural que, durante o período, adotem-se atitudes precavidas quanto ao risco de efeitos tóxicos ao embrião e ao feto ${ }^{19}$. O conhecimento dos riscos traz mais segurança.

A programação de medicamentos (Tabela 1) prevê o seu fornecimento contínuo e racional. Ela pode ser desenvolvida com base no perfil epidemiológico do município ou do seu consumo histórico, que foi a opção de Praia Grande. O uso exclusivo de um único critério técnico pode permitir a manutenção de erros contínuos de fornecimento, daí a necessidade de conjugar várias estratégias 5 . $\mathrm{O}$ consumo é ajustado mensalmen- te conforme a planilha realizada pelas unidades. Porém, a falta de informatização no controle de estoque assim como o rodízio de funcionários são fatores que podem levar a erros neste consumo histórico, o qual pode estar espelhando uma contínua privação no acesso aos medicamentos.

A compra de medicamentos no município se dá por pregão presencial, em que os fornecedores apresentam lances em sessão pública no sentido contrário ao leilão comum, ou seja, em valores decrescentes a partir de um preço máximo de referência. É um procedimento legal que simplifica, possibilitando a redução dos custos operacionais e dos preços efetivamente contratados. Contudo, o pregão ocorre duas vezes ao ano, freqüência superior à ideal, pois compras anuais consolidadas aumentam as possibilidades de redução dos preços em decorrência do volume maior 5 .

Não há manutenção da regularidade no abastecimento, dado que a falta de critérios mais abrangentes para fazer a programação de medicamentos leva à necessidade de compras emergenciais, como foi constatado.

Existem duas características administrativas no Município de Praia Grande que podem contribuir para as deficiências na programação: a falta de treinamento dos funcionários no controle de estoque e a ausência de funcionários fixos. O rodízio de auxiliares de enfermagem nas farmácias das unidades pode levar a falta de vínculo e descompromisso com a função.

A aquisição de medicamentos exige o estabelecimento de critérios para os fornecedores, as questões técnicas deveriam se conjugar ao custo, numa adequada relação custo/benefício. Em Praia Grande, a escolha do fornecedor limita-se às exigências legais 20 , não se exigindo a caracterização da competência técnica no fabrico do medicamento. Dentro dessa linha de pensamento, foi constatado que há a opção pela compra de medicamentos similares, se atendidas as exigências de preço.

A compra de medicamentos similares fere a legislação vigente, que impõe a obrigatoriedade da prescrição em denominação comum brasileira, e a oferta do medicamento genérico ou o de referência 21 .

Ao serem analisadas as condições de armazenamento como outro parâmetro da assistência farmacêutica (Tabela 3), observou-se que, de uma maneira geral, a situação encontrada era adequada com controle informatizado de estoque, boa ventilação e luminosidade. $\mathrm{O}$ ambiente mostrou-se limpo, com coletores de lixo tampados, sem apresentar poeira, nem indícios de roedores e aves. A localização era boa, de fácil acesso e com espaço suficiente para movimentação de 
veículos. Os medicamentos estavam colocados sobre estrados de madeira evitando contato direto com o chão e ausência de empilhamento excessivo de caixas. Os medicamentos com prazo de validade mais próxima estavam dispostos de modo a serem distribuídos primeiro. Os corredores entre os produtos permitiam livre trânsito. E não foram observadas irregularidades quanto à segurança e à dimensão da Central de Abastecimento Farmacêutico. Os medicamentos controlados pela Portaria no. 344/98 22 (nos quais se incluem, entre outros, os medicamentos psicoativos) são estocados isoladamente dos demais, sob controle e responsabilidade do farmacêutico, de acordo com a exigência da legislação.

Entretanto, constataram-se sinais de umidade nas paredes e algumas embalagens estavam sujas, com aspecto semelhante ao de algum produto derramado. A temperatura ambiente era controlada diariamente, no entanto não havia um sistema de refrigeração adequado, atingindo-se, portanto, temperaturas acima do ideal para uma Central de Abastecimento Farmacêutico. Outro aspecto observado que não favorece a organização da Central de Abastecimento Farmacêutico é o fato de armazenarem, no mesmo ambiente físico, outros produtos que não sejam medicamentos, tais como materiais de limpeza e de escritório.

Um problema constatado tanto na Central de Abastecimento Farmacêutico quanto nas unidades de atendimento, que pode comprometer a qualidade da assistência farmacêutica, é o fato de não existir restrição à entrada de pessoas não lotadas no setor.

Apesar de ser uma atividade simples, o armazenamento exige padronização de procedimentos 5. Tais normas não foram encontradas em Praia Grande. Há de se ressaltar o fato de não haver normas escritas para a destruição de medicamentos vencidos.

O medicamento é um bem físico que necessita de cuidados com compra e armazenamento. Por outro lado é um instrumento terapêutico que demanda necessidades especiais para o seu uso racional. O uso do medicamento, principalmente pela gestante, merece especial atenção pelos riscos potenciais ao feto em desenvolvimento. A investigação da dispensação, incluindo-se orientações gerais sobre o uso de medicamentos, pode ampliar a margem de segurança, visto que a gravidez é uma ocasião única, e a exposição de um afeta dois organismos 23,24,25.

Ao realizar as visitas às unidades básicas de saúde e de saúde da família não foram constatadas diferenças significativas entre as mesmas em relação aos parâmetros analisados. Observou-se (Tabela 4) a ausência do profissional farmacêuti- co e deve-se considerar que, por força da legislação 26 , a dispensação deveria ser desempenhada exclusivamente por esse profissional. Nas unidades investigadas, a dispensação ficava sob a responsabilidade de auxiliares de enfermagem que na maioria das vezes se revezam na função, não existindo um profissional fixo. Além disso, nenhum funcionário havia participado de algum processo de treinamento.

A importância da presença do farmacêutico pode ser atestada por trabalhos como os desenvolvidos por Rúa \& Tolosa 27 que verificaram que cerca de $14 \%$ das prescrições analisadas apresentavam problemas relacionados aos medicamentos, dos quais mais de um terço foram solucionados sob intervenção direta do farmacêutico. E ainda, Romano-Lieber et al. ${ }^{28}$, em revisão de estudos sobre a intervenção dos farmacêuticos em idosos, concluem que a ação desse profissional pode reduzir custos, interagir com o prescritor, melhorando a qualidade da prescrição, e promover maior adesão ao tratamento. Outros estudos também corroboram o incremento da qualidade da farmacoterapia mediante ação específica do farmacêutico 29,30,31.

Em relação aos serviços públicos, os governos e dirigentes discutem a questão do abastecimento de medicamentos e as estratégias de financiamento, mas geralmente não se preocupam com a estruturação e organização deste serviço ${ }^{5}$. A maioria das farmácias de unidades básicas de saúde funciona porque um leigo ou profissional com parcos conhecimentos sobre medicamentos atua na dispensação ${ }^{32}$.

O controle de saída dos medicamentos era realizado de maneira informatizada em apenas $30 \%$ das unidades. Um sistema de controle de estoque não informatizado contribui desfavoravelmente para o gerenciamento dos investimentos de reposição e no levantamento dos dados necessários à tomada de decisão, pois demanda muito tempo e pode não apresentar os estoques disponíveis em tempo hábil, bem como não apresenta confiabilidade suficiente em decorrência da possibilidade de ocorrer equívoco quando da efetivação de seus registros 33 .

Em relação ao acesso a medicamentos, observou-se que em aproximadamente $85 \%$ das unidades ocorria a carência de medicamentos usualmente prescritos para gestante. A falta freqüente do ácido fólico e $\alpha$-metil-dopa, por exemplo, foi citada, respectivamente, em $65 \%$ e $30 \%$ das entrevistas realizadas.

A carência do ácido fólico é o mais importante fator de risco para os defeitos do tubo neural que, por sua vez, são importantes causas de natimortalidade fetal 34,35. A suplementação periconcepcional durante o primeiro trimestre 
de gravidez tem reduzido os riscos em cerca de $50 \%$ a $70 \% 36,37,38$. Resultados recentes colocam o Brasil no patamar dos países com as mais altas taxas de defeitos do tubo neural, todavia as prevalências são subestimadas; muitas gestações são naturalmente ou deliberadamente interrompidas $38,39,40$.

A $\alpha$-metil-dopa é um dos anti-hipertensivos mais utilizados pelas gestantes e tem como objetivo evitar as complicações decorrentes de síndromes hipertensivas como a prematuridade e morbi-mortalidade perinatal devido à hipoxia intra-uterina 41,42 .

A falta de medicamentos tem duas vertentes a serem consideradas. Uma é a falta em si, que é preocupante, já que as pacientes eram orientadas a procurar o medicamento em outra unidade do município, fato que poderia gerar uma peregrinação da gestante, pois a falta do medicamento ocorria na maioria das unidades. A outra faceta a ser desvelada é a pouca importância dada à falta de medicamentos, considerando esse fato como normal. Em relatos dos funcionários, como a falta sendo "somente no final do mês" (grifo nosso) poderia ser menos importante do que a falta em outro dia qualquer. Parece óbvio que ou o medicamento falta ou não.

\section{Considerações finais}

A assistência farmacêutica mantém-se fragmentada no Sistema Único de Saúde (SUS), o que poderia ser revertido com a adoção de um enfoque sistêmico na sua organização. Há de se criar relações entre todos os setores envolvidos, estabelecendo-se fluxos em um conjunto articulado 5 . Por exemplo, no caso da Comissão de Farmácia e Terapêutica, se os municípios não têm estrutura para compor comissões municipais, por que não propor comissões regionais, na forma de consórcio, incluídas nos processos de pactuação?

Os mecanismos de compra utilizados, embora legais, podem levar a que sejam dispensados medicamentos sem comprovada bioequivalên- cia, na opção pelos similares em detrimento dos genéricos. A opção pelos similares se dá em função de, em alguns casos, apresentarem preços menores.

Cabe lembrar que a assistência farmacêutica é o termo utilizado na Política Nacional de Medicamentos 11 para designar o grupo de atividades relacionadas com o medicamento, destinadas a apoiar as ações de saúde demandadas por uma comunidade. Em relação à gestante, a assistência farmacêutica ainda precisa encontrar meios de se aperfeiçoar e contribuir de maneira mais efetiva com a saúde da população.

O medicamento na assistência à saúde sofre de um paradoxo, pela aparente contradição existente entre a importância dada à aquisição dos medicamentos sem a contrapartida do seu uso racional e adequado. Ao mesmo tempo em que há uma medicalização da saúde, dando-se uma importância maior à terapêutica do que à promoção da saúde, por outro lado o uso racional da farmacoterapia é deixado de lado. Essa assertiva vem do fato de que o medicamento é simplesmente entregue ao usuário, sem orientações importantes sobre o seu uso. A falta de formação continuada da equipe de saúde em relação ao uso racional do medicamento pode ser uma das causas que leva o medicamento a ser relacionado em cerca de $34 \%$ dos eventos de intoxicação no Estado de São Paulo 43 e em 28\% dos casos registrados anualmente no Brasil 44. Enquanto nos Estados Unidos as reações adversas aos medicamentos situam-se entre a quarta ou sexta causa de morte 25 .

Sem dúvida, nos últimos anos, houve um crescimento importante na oferta de serviços no nível primário de atenção no Brasil. No entanto, os resultados encontrados na presente investigação sugerem que, para o aperfeiçoamento desta política, garantida a ampliação do acesso, a eqüidade e a integralidade, a avaliação da assistência farmacêutica deve ser incorporada cotidianamente, tanto na elaboração quanto na execução e avaliação das políticas de atenção básica implementadas em nosso país. 


\section{Resumo}

O uso de medicamentos na atenção primária à saúde vem crescendo continuadamente nas últimas décadas, de tal modo que avaliar a assistência farmacêutica prestada aos pacientes nesse nível de atenção adquire relevância. O objetivo deste artigo é avaliar a assistência farmacêutica ofertada na rede básica de saúde em município da Região Metropolitana da Baixada Santista, Estado de São Paulo, Brasil, delimitando-se para a análise a saúde da mulher, especialmente no período gestacional. Foram realizadas: análise de dados secundários; análise documental; entrevistas com atores-chave; visita aos serviços de saúde e à Central de Abastecimento Farmacêutico. Foram encontradas inadequações em praticamente todas as etapas - seleção, programação, aquisição, armazenamento, distribuição e dispensação - da assistência farmacêutica. Os resultados encontrados sugerem que para o aperfeiçoamento da atenção primária à saúde, garantida a ampliação do acesso, a equidade e a integralidade, a avaliação da assistência farmacêutica deveria ser incorporada cotidianamente.

Assistência Farmacêutica; Atenção Primária à Saúde; Avaliação de Serviços de Saúde; Cuidado Pré-Natal

\section{Referências}

1. Department of Health. Statistics of prescriptions dispensed in the community - England 1992 to 2002. http://www.dh.gov.uk/en/ (acessado em 20/ Jan/2007)

2. Starfield B. Atenção primária: equilíbrio entre necessidades de saúde, serviços e tecnologia. Brasília: Organização das Nações Unidas para a Educação, a Ciência e a Cultura/Ministério da Saúde; 2004.

3. Bermudez JAZ, Bonfim JRA, organizadores. Medicamentos e a reforma do setor saúde. São Paulo: Editora Hucitec/Sociedade Brasileira de Vigilância de Medicamentos; 1999.

4. Lugones R. Analisis comparado de política de medicamentos. In: Bermudez JAZ, Bonfim JRA organizadores. Medicamentos e a reforma do setor saúde. São Paulo: Editora Hucitec/Sociedade Brasileira de Vigilância de Medicamentos; 1999. p. 79-92.

5. Marin N, organizador. Assistência farmacêutica para gerentes municipais. Rio de Janeiro: Organização Pan-Americana da Saúde/Organização Mundial da Saúde; 2003.

6. Cosendey MAE, Hartz ZMA, Bermudez JAZ. Validation of a tool for assessing the quality of pharmaceutical services. Cad Saúde Pública 2003; 19:395406.

\section{Colaboradores}

A. Bousquat participou do delineamento do estudo, da análise de dados e coordenou a discussão dos resultados e a organização e redação final deste artigo. M. R. S. Vieira e P. A. Lorandi colaboraram na elaboração do instrumento, acompanharam a coleta e a análise de dados e contribuíram para a discussão e elaboração do artigo.

\section{Agradecimentos}

Esta investigação faz parte do projeto de pesquisa $\mathrm{Ca}$ racterização da Mortalidade Neonatal e Perinatal na Região Metropolitana da Baixada Santista, financiada pelo Departamento de Ciência e Tecnologia em Saúde do Ministério da Saúde/Conselho Nacional de Desenvolvimento Científico e Tecnológico (MS-DECIT/CNPq, processo 403593/2004-2).
7. Brasil. Portaria no. 399/GM. Divulga o pacto pela saúde 2006 - consolidação do SUS e aprova as diretrizes operacionais do referido pacto. Diário Oficial da União 2006; 23 fev.

8. Lopes EZ. A implantação do Programa Saúde da Família em Praia Grande, São Paulo [Dissertação de Mestrado]. Santos: Universidade Católica de Santos; 2005.

9. Secretaria de Atenção à Saúde, Ministério da Saúde. Política nacional de atenção integral à saúde da mulher: princípios e diretrizes. Brasília: Ministério da Saúde; 2004.

10. Viana ALA, organizador. Caracterização dos municípios paulistas com população superior a $100 \mathrm{mil}$ habitantes. São Paulo: Centro de Estudos de Cultura Contemporânea/Consórcio Medicina USP; 2006.

11. Departamento de Atenção Básica, Secretaria de Políticas de Saúde, Ministério da Saúde. Política nacional de medicamentos. Brasília: Ministério da Saúde; 2001.

12. Secretaria de Atenção à Saúde, Ministério da Saúde. Pré-natal e puerpério: atenção qualificada e humanizada - manual técnico. Brasília: Ministério da Saúde; 2005.

13. Secretaria de Políticas de Saúde, Ministério da Saúde. Relação nacional de medicamentos essenciais - RENAME. 3a Ed. Brasília: Ministério da Saúde; 2002. 
14. Departamento de Atenção Básica, Secretaria de Políticas de Saúde, Ministério da Saúde. Assistência farmacêutica na atenção básica: instruções técnicas para sua organização. Brasília: Ministério da Saúde; 2001.

15. Silveira DS, Santos IS, Costa JSD. Atenção pré-natal na rede básica: uma avaliação da estrutura e do processo. Cad Saúde Pública 2001; 17:131-9.

16. Koffman MD, Bonadio IC. Avaliação da atenção pré-natal em uma instituição filantrópica da cidade de São Paulo. Rev Bras Saúde Matern Infant 2005; 5:23-32.

17. Secretaria Municipal da Saúde de São Paulo. REMUME. São Paulo: Secretaria Municipal da Saúde de São Paulo; 2004.

18. Guerra Jr. AA, Acúrcio FA, Gomes CAP, Miralles M, Girardi SN, Werneck GAF, et al. Disponibilidade de medicamentos essenciais em duas regiões de Minas Gerais, Brasil. Rev Panam Salud Pública 2004; 15:168-75.

19. Fonseca MRCC, Fonseca E, Bergsten-Mendes G. Prevalência do uso de medicamentos na gravidez: uma abordagem farmacoepidemiológica. Rev Saúde Pública 2002; 36:205-12.

20. Brasil. Lei ${ }^{\circ}$. 8.666. Regulamenta o art. 37, inciso XXI, da Constituição Federal, institui normas para licitações e contratos da administração pública e dá outras providências. Diário Oficial da União 1994; 6 jul.

21. Brasil. RDC $n^{\circ}$. 16. Aprova o regulamento técnico para medicamentos genéricos, anexo I. Diário Oficial da União 2007; 2 mar.

22. Brasil. Portaria no ${ }^{\circ}$. 344. Aprova o regulamento técnico sobre substâncias e medicamentos sujeitos a controle especial. Diário Oficial da União 1999; 1 fev.

23. Gomes KRO, Moron AF, Silva RS, Siqueira AAF. Prevalência do uso de medicamentos na gravidez e relações com as características maternas. Rev Saúde Pública 1999; 33:246-54.

24. Lagoy CT, Joshi N, Cragan JD, Rasmussen SA. Medication use during pregnancy and lactation: an urgent call for public health action. J Womens Health 2005; $14: 104-9$

25. Karrer M, Buttet P, Vincent I, Ferron C. Comment pratiquer l'éducation pour la santé en médecine générale ou en pharmacie? Modalités de mise en oeuvre d'une expérimentation auprès de femmes enceintes, d'adolescents ou de personnes âgées. Santé Publique 2004; 16:471-85.

26. Brasil. Lei $n^{\circ}$. 5.991. Dispõe sobre o controle sanitário do comércio de drogas, medicamentos, insumos farmacêuticos e correlatos, e dá outras providências. Diário Oficial da União 1973; 19 dez.

27. Rúa EP, Tolosa JLS. Efectividad y estimación de costes en una intervención sobre problemas relacionados con los medicamentos en atención primaria. Aten Primaria 2005; 35:472-7.

28. Romano-Lieber NS, Teixeira JJV, Farhat FCLG, Ribeiro E, Crozatti MTL, Oliveira GSA. Revisão dos estudos de intervenção do farmacêutico no uso de medicamentos por pacientes idosos. Cad Saúde Pública 2002; 18:1499-507.
29. Andrade MA, Silva MVS, Freitas O. Assistência farmacêutica como estratégia para o uso racional de medicamentos em idosos. Semina 2004; 25:55-63.

30. Lyra Júnior DP, Amaral RT, Veiga EV, Cárnio EC, Nogueira MS, Pelá IR. A farmacoterapia no idoso: revisão sobre a abordagem multiprofissional no controle da hipertensão arterial sistêmica. Rev Latinoam Enferm 2006; 14:435-41.

31. Hassell K, Noyce PR, Rogers A, Harris J, Wilkinson J. A pathway to the GP: the pharmaceutical "consultation" as a first port of call in primary health care. Fam Pract 1997; 14:498-502.

32. Viera FS. Possibilidades de contribuição do farmacêutico para a promoção da saúde. Ciênc Saúde Coletiva 2007; 12:213-20.

33. Pinheiro ACM. Gerenciamento de estoque farmacêutico. Revista Eletrônica de Contabilidade 2005; 1:80-94.

34. Cunha CJ, Fontana T, Garcias GL, Martino Roth MG. Fatores genéticos e ambientais associados à espinha bífida. Rev Bras Ginecol Obstet 2005; 27:268-74.

35. Goh YI, Bollano E, Einarson TR, Koren G. Prenatal multivitamin supplementation and rates of congenital anomalies: a meta-analysis. J Obstet Gynaecol Can 2006; 28:680-9.

36. Santos LMP, Pereira MZ. Efeito da fortificação com ácido fólico na redução dos defeitos do tubo neural. Cad Saúde Pública 2007; 23:17-24.

37. Botto LD, Olney RS, Erickson JD. Vitamin supplements and the risk for congenital anomalies other than neural tube defects. Am J Med Genet C Semin Med Genet 2004; 125:12-21.

38. Lima HT, Saunders C, Ramalho A. Ingestão dietética de folato em gestantes do município do Rio de Janeiro. Rev Bras Saúde Matern Infant 2002; 2:303-11.

39. Castilla EE, Orioli IM, Lopez-Camelo JS, Dutra MG, Nazer-Herrera J. Preliminary data on changes in neural tube defects prevalence rates after folic acid fortification in South America. Am J Med Genet A 2003; 123:123-8.

40. World Health Organization. World atlas of birth defects (2003). http://www.who.int/genomics/publi cations/en/ (acessado em 10/Set/2007).

41. Ferrão MHL, Pereira ACL, Gersgorin HCTS, Paula TAA, Corrêa RRM, Castro ECC. Efetividade do tratamento de gestantes hipertensas. AMB Rev Assoc Med Bras 2006; 52:390-4.

42. Sibai BM. Diagnosis and management of gestational hipertension and preeclampsia. Obstet Gynecol 2003; 102:181-92.

43. Gandolfi E, Andrade MGG. Eventos toxicológicos relacionados a medicamentos no Estado de São Paulo. Rev Saúde Pública 2006; 40:1056-64.

44. Sistema Nacional de Informações Tóxico-Farmacológicas. Medicamentos. http://www.fiocruz. $\mathrm{br} /$ sinitox/medicamentos.htm (acessado em 24/ Abr/2007)

Recebido em 15/Jun/2007

Versão final reapresentada em 02/Out/2007 Aprovado em 08/Out/2007 